\title{
CD147 mediates transforming growth factor-ß1-induced epithelial-mesenchymal transition and cell invasion in squamous cell carcinoma of the tongue
}

\author{
SHINSUKE SUZUKI, SATOSHI TOYOMA, TADAHIRO TSUJI, YOHEI KAWASAKI and TAKECHIYO YAMADA
}

\author{
Department of Otorhinolaryngology, Head and Neck Surgery, Akita University \\ Graduate School of Medicine, Akita 010-8543, Japan
}

Received July 28, 2018; Accepted December 28, 2018

DOI: $10.3892 /$ etm.2019.7230

\begin{abstract}
Epithelial-mesenchymal transition (EMT) is a physiological process in which epithelial cells attain the motile and invasive characteristics of mesenchymal cells, which results in the development of increased migratory and invasive cell behavior, serving as a vital mechanism of cancer progression. Hence, controlling the EMT for cancer treatment, including head and neck squamous cell carcinoma (HNSCC), is imperative. Among EMT-associated factors, transforming growth factor- $\beta$ (TGF- $\beta$ ) is a well-established potent inducer. Recent research has revealed that CD147, a member of the immunoglobulin superfamily, promotes the EMT. However, the role of CD147 in the EMT and the following tumorigenicity in HNSCC has not been completely elucidated. This study aims to investigate the role of CD147 in the EMT and related tumorigenicity in HNSCC. The present study used two HNSCC cell lines, SAS and FaDu, for in vitro studies. In HNSCC cells, TGF- $\beta 1$ induced spindle-shaped morphological changes, and western blot analysis revealed that TGF- $\beta 1$ induced changes in EMT markers, downregulation of vimentin, and upregulation of E-cadherin, yet increased CD147. In addition, TGF- $\beta 1$ increased cell migration in HNSCC cells. However, a TGF- $\beta 1$-induced alteration in EMT makers was attenuated with CD147 silencing by small interfering RNA (siRNA) in SAS cells. In addition, the TGF- $\beta 1$-induced cell invasion of SAS was attenuated with CD147 silencing. In conclusion, the
\end{abstract}

Correspondence to: $\mathrm{Dr}$ Shinsuke Suzuki, Department of Otorhinolaryngology, Head and Neck Surgery, Akita University Graduate School of Medicine, 1-1-1 Hondo, Akita 010-8543, Japan

E-mail:suzukis@med.akita-u.ac.jp

Abbreviations: DMEM, Dulbecco's modified Eagle's medium; EMMPRIN, extracellular matrix metalloproteinase inducer; EMT, epithelial-mesenchymal transition; HNSCC, head and neck squamous cell carcinoma; RNA, ribonucleic acid; SCC, squamous cell carcinoma; $\mathrm{SD}$, standard deviation

Key words: CD147, epithelial-mesenchymal transition, head and neck cancer, transforming growth factor- $\beta 1$, invasion, migration present study suggests that CD147 mediates TGF- $\beta 1$-induced EMT and tumorigenicity in HNSCC. Hence, CD147 may serve as a vital therapeutic target in HNSCC.

\section{Introduction}

Head and neck squamous cell carcinoma (HNSCC) is the leading histology-type malignancy in the upper aerodigestive tract and is characterized by a marked propensity of invasion and cervical lymph node metastasis associated with poor outcomes. Despite advancements in multimodality therapy surgery, radiation, chemotherapy, and recent progress in immunotherapy, HNSCC often remains fatal, necessitating new and more efficacious therapeutic strategies to enhance the HNSCC survival (1).

Epithelial-mesenchymal transition (EMT) is a biological process that allows a polarized epithelial cell, which normally interacts with basement membrane via its basal surface, to undergo multiple biochemical changes that enable it to assume a mesenchymal cell phenotype. These changes include enhanced migratory capacity, invasiveness, increased resistance to apoptosis, and a considerably increased production of extracellular matrix components. Hence, EMT is considered a vital mechanism of cancer progression, particularly in solid tumors. Different biomarkers have been used to present EMT. The upregulation of mesenchymal cell markers (e.g., fibronectin, vimentin, $\alpha$-smooth muscle actin, and $\mathrm{N}$-cadherin) and the downregulation of epithelial markers (e.g., E-cadherin, ZO-1, and $\alpha$ - and $\gamma$-catenin) are the biomarkers often used in monitoring the progression of EMT in individuals with progressive cancer (2).

Transforming growth factor- $\beta$ (TGF- $\beta$ ) is a pleiotropic cytokine involved in various activities, including differentiation, growth, apoptosis, inflammation, tissue remodeling, and wound healing (3). TGF- $\beta$ can initiate and maintain EMT via different biological systems and pathophysiological contexts. TGF- $\beta$-derived signals can coordinate the expression and function of Snail, ZEB, and bHLH factors and promote their interplay in EMT and migration of malignant tumor (4). Among the TGF- $\beta$ subtypes, TGF- $\beta 1$ was first considered an EMT inducer in normal mammary epithelial cells (5); since then, it has been believed that TGF- $\beta 1$ mediates EMT in cancer 
cells and HNSCC (6). Therefore, in cancer, the mechanisms of EMT have gradually been elucidated over the years.

CD147, also termed as the extracellular matrix metalloproteinase inducer (EMMPRIN), is a member of the immunoglobulin superfamily, and it is highly expressed in cancer cells. Moreover, it is known to cause different malignancies, including HNSCC $(7,8)$.

To prevent the progression of malignant tumors, several studies have attempted to reveal the mechanisms underlying CD147-induced tumorigenicity in various cancer types $(9,10)$. The number of studies regarding the contribution of CD147 to HNSCC progression is also increasing. We have previously reported that CD147 increased cell invasiveness, proliferation, and drug resistance via interactions with its ligand and cyclophilin A in individuals with HNSCC (11). In addition, CD147 expression correlates with lymph node metastasis in squamous cell carcinoma of the tongue (12). However, the role of CD147 in tumorigenicity in HNSCC and the underlying mechanism are not completely understood. Therefore, further analysis must be conducted.

Recently, CD147 has been found to promote EMT in certain individuals with solid tumors $(9,13)$. However, its role in EMT in individuals with HNSCC remains unclear. Therefore, the present study aimed to elucidate the role of CD147 in EMT and tumorigenicity in HNSCC.

\section{Materials and methods}

Cells and cell culture. We purchased SAS, a human tongue squamous cell carcinoma cell line, from the RIKEN Cell Bank (Tsukuba, Japan). FaDu cells, a human hypopharyngeal squamous cell carcinoma cell line, were kindly gifted by the Department of Cell Biology and Morphology, Akita University Graduate School of Medicine (Akita, Japan); both cell lines were used for in vitro studies. All cells were maintained in the Dulbecco's modified Eagle's medium (DMEM; Merck KGaA, Darmstadt, Germany) supplemented with $10 \%$ fetal bovine serum in a humidified atmosphere containing $5 \% \mathrm{CO}_{2}$ at $37^{\circ} \mathrm{C}$. For stimulation experiments, we preincubated SAS and FaDu cells with serum-free DMEM and subsequently incubated with serum-free medium containing 10 or $20 \mathrm{ng} / \mathrm{ml}$ of TGF- $\beta 1$ (Wako, Osaka, Japan).

Immunoblotting. Protein expression was detected by western blot analysis using actin as an internal control. We lysed cell lines in detergent containing $1 \% \mathrm{NP} 40,150 \mathrm{mmol} / \mathrm{l} \mathrm{NaCl}$, $1 \mathrm{mmol} / 1$ EDTA, $0.1 \mathrm{mmol} / 1$ phenylmethylsulfonyl fluoride, $1 \mu \mathrm{g} / \mathrm{ml}$ leupeptin, and $1 \mu \mathrm{g} / \mathrm{ml}$ aprotinin and determined the protein levels using the Bio-Rad Protein Assay Method (Bio-Rad Laboratories Inc., Hercules, CA, USA). Then, we separated $40 \mu \mathrm{g}$ of the total protein on $8 \%$ SDS-PAGE gels and transferred it to nitrocellulose membranes using a semidry transfer machine (Bio-Rad Laboratories, Inc.). Next, we blocked membranes with $5 \%$ skimmed milk/TBS with Tween-20 solution for $1 \mathrm{~h}$ at room temperature, incubated with primary antibodies in 5\% skimmed milk in TBS-T overnight at $4^{\circ} \mathrm{C}$. After washing with TBS-T three times, the membranes were incubated for $1 \mathrm{~h}$ with horseradish-peroxidase-conjugated secondary antibody (Bio-Rad Laboratories, Inc.) 1:3,000 diluted in $5 \%$ skimmed milk in TBS-T. Then, we rinsed the filters with TBS-T three times and developed the blot using Luminol Reagent (Santa Cruz Biotechnology, Inc., Santa Cruz, CA, USA) by autoradiography. In this study, we used the following primary antibodies: rabbit anti-CD147, rabbit anti-E-cadherin, goat anti-vimentin (1:1,000; Santa Cruz Biotechnology, Inc.), and mouse anti- $\beta$-actin (1:5,000; Merck Millipore, Tokyo, Japan).

Wound-healing assay. We conducted the wound-healing assay in six-well tissue culture plates. In addition, we cultured SAS and FaDu cells as a confluent monolayer. Then, the medium was changed to serum-free, and after $24 \mathrm{~h}$, a cell-free area was created by gently scratching the cell monolayer with a sterile $10-\mu 1$ pipette tip, resulting in the creation of a $1-\mathrm{mm}$-wide cell-free area. Immediately after scratching, the medium was replaced with a fresh medium or a medium containing $10 \mathrm{ng} / \mathrm{ml}$ of TGF- $\beta 1$. The same wound areas were observed and photographed under an inverted microscope (Olympus, Tokyo, Japan), and the distance of the scratch closure was examined at 0 and $18 \mathrm{~h}$.

Small interfering RNA (siRNA) and siRNA transfection. CD147 siGENOME siRNA (Dharmacon RNA Technologies, Lafayette, CO) was transfected into SAS cells for CD147 silencing. We used the siGENOME nontargeting siRNA as control. Furthermore, siRNA transfections were performed using Lipofectamine 2000 (Thermo Fisher Scientific, Inc.). In brief, $1.8 \times 10^{5}$ of SAS cells were plated on 6 well plate. After $24 \mathrm{~h}$ incubation in complete media, cells were transfected with 200 pmol of CD147 siRNA or nontargeting control siRNA. The transfection medium was replaced with complete media after $4 \mathrm{~h}$ of transfection.

Matrigel invasion assay. We evaluated cell invasiveness in vitro using Matrigel-coated semipermeable-modified Boyden inserts with a pore size of $8 \mu \mathrm{m}$ (BD Biosciences, Franklin Lakes, NJ, USA). In addition, SAS and FaDu cells were plated at a density of $2.5 \times 104$ cells/insert in serum-free medium with or without TGF- $\beta$ (10 ng/ml). Notably, the lower chamber contained DMEM + 10\% FBS and served as a chemoattractant. Meanwhile, we plated cells in 96-well plates to serve as loading controls. After 48 -h treatment at $37^{\circ} \mathrm{C}$ in a $5 \% \mathrm{CO}_{2}$ incubator, we removed the cells in the insert by wiping gently with a cotton swab. Next, cells on the reverse side of the insert were fixed and stained using Diff-Quick (Sysmex, Kobe, Japan) according to the manufacturer's instructions. We counted the invading cells in four representative fields using light microscopy at magnification, $\mathrm{x} 200$. Moreover, we evaluated mean \pm standard deviation (SD) from three independent experiments. Furthermore, the cells plated on the 96-well plate were assessed using a 3-(4,5-dimethylthiazol-2-yl)-2,5-diphenyltetrazolium bromide assay to determine the metabolically active cells. Of note, the number of invading cells was adjusted accordingly.

Statistical analysis. In this study, we used the Wilcoxon-Mann-Whitney two-tailed exact test (Statcel 3; OMS Publishing, Tokorozawa, Japan) to assess the statistical significance of the differences in the wound closure, protein expression, and invasion studies. Data are presented as mean \pm SD from experiments that were repeated at least three 
A

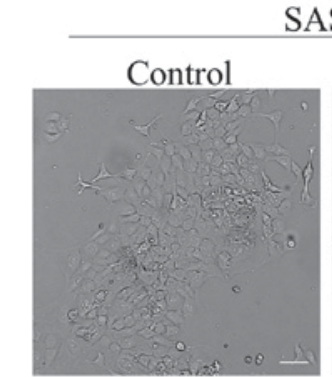

SAS

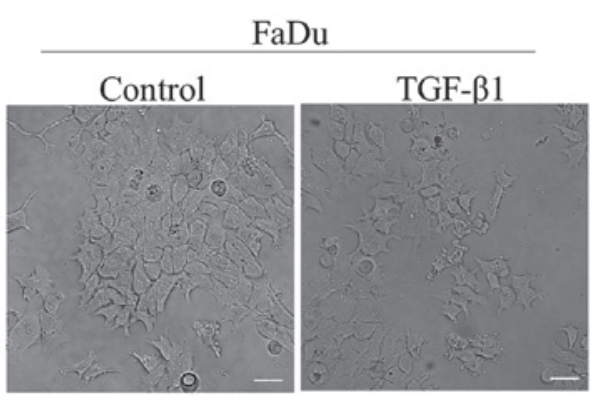

B

SAS
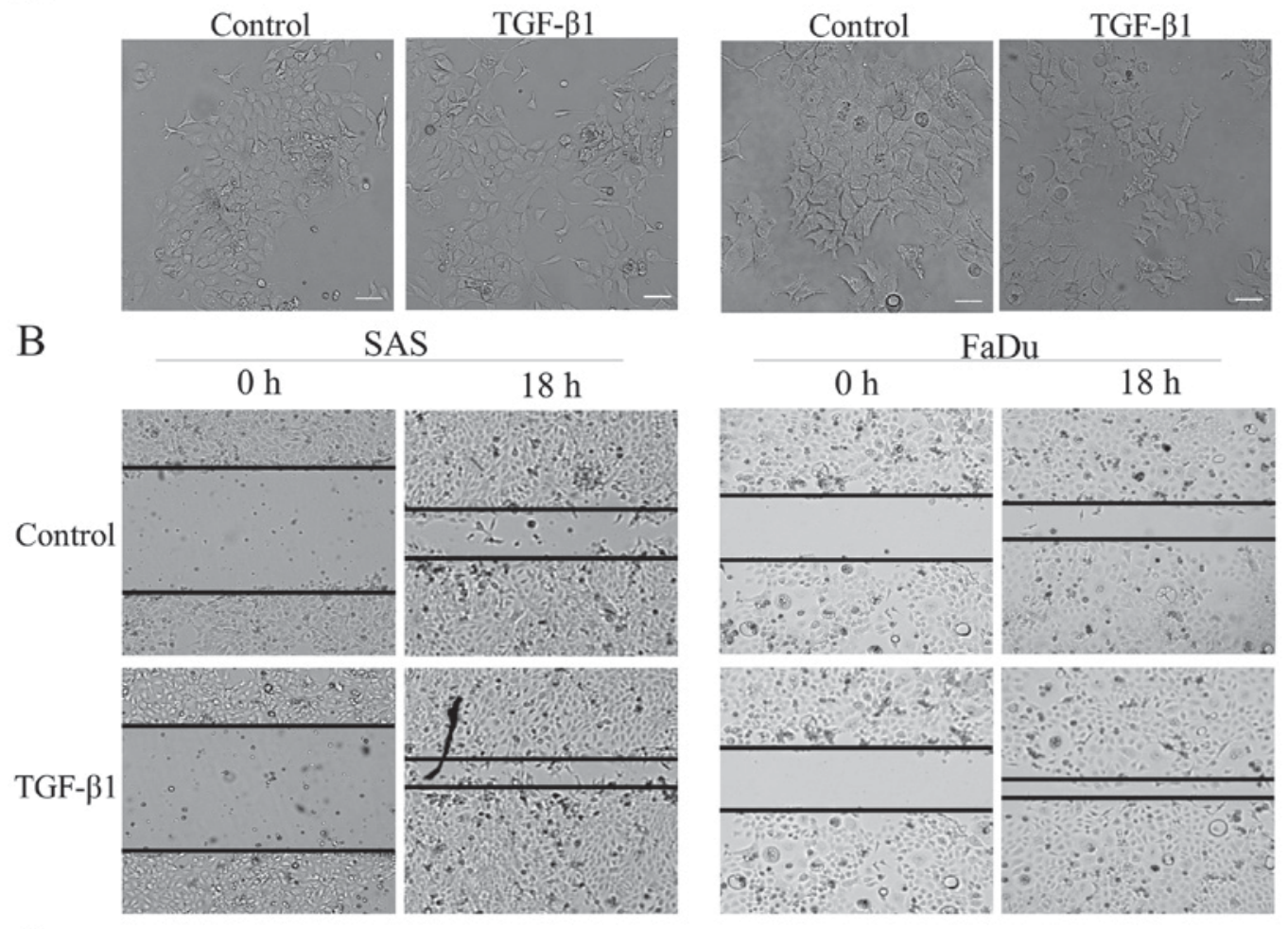

$\mathrm{C}$
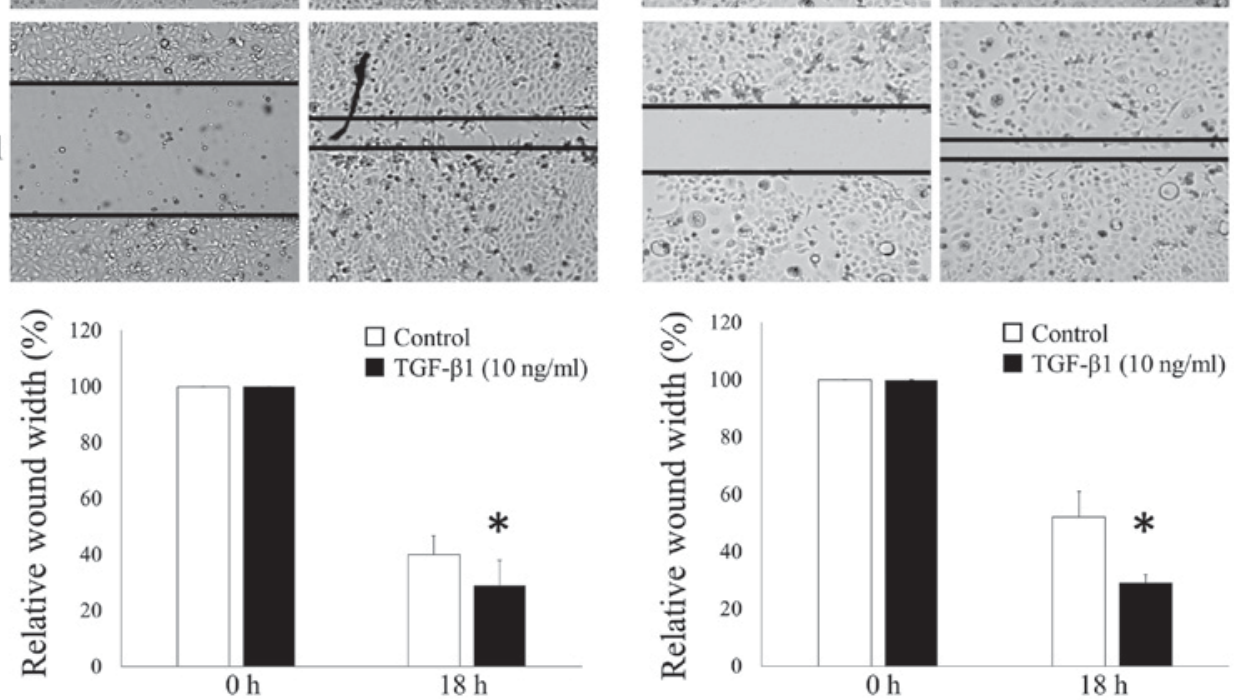

$\square$ Control

- TGF- $\beta 1(10 \mathrm{ng} / \mathrm{ml})$

$0 \mathrm{~h}$
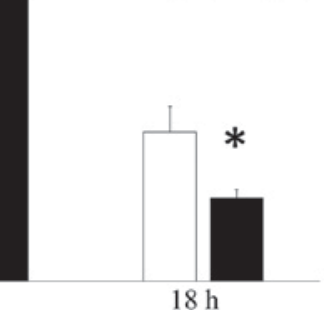

Figure 1. TGF- $\beta 1$ induces the morphological change and the cell migration of HNSCC cells. SAS and FaDu cells were incubated with $20 \mathrm{ng} / \mathrm{ml}$ of TGF- $\beta$ for 48 h. (A) SAS and FaDu cells displayed a cobblestone appearance and growth in clusters (control); SAS and FaDu cells lost adhesiveness and exhibited a spindle-shaped morphology (TGF- $\beta 1$ ). The cells were examined using phase-contrast microscopy (scale bar, $100 \mu \mathrm{m}$ ). (B) The cell migration of HNSCC cells was assessed by the wound-healing assay. SAS and FaDu cells were scratched by a sterile $10-\mu l$ pipette tip, followed by the PBS treatment or $10 \mathrm{ng} / \mathrm{ml}$ of TGF- $\beta 1$ for $18 \mathrm{~h}$. TGF- $\beta 1$ increases healing of the scratched cells (original magnification, $\mathrm{x} 40$ ). (C) Data shown are the means of three measurements, and the bars represent the SD of the mean. ${ }^{*} \mathrm{P}<0.05$ vs. control at $18 \mathrm{~h}$. TGF- $\beta 1$, transforming growth factor- $\beta 1$; HNSCC, head and neck squamous cell carcinoma.

times. $\mathrm{P}<0.05$ was considered to indicate a statistically significant difference.

\section{Results}

Induction of the morphological change and the cell migration of HNSCC cells by TGF- $\beta 1$. We cultured two HNSCC cell lines, SAS and FaDu cells, with TGF- $\beta 1$ to investigate whether TGF- $\beta 1$ induced the EMT-related phenomena in HNSCC. We examined the morphological change using phase-contrast microscopy. Both cell lines exhibited a cobblestone appearance and clustered growth. However, cells lost adhesiveness and displayed a spindle-shaped morphology with TGF- $\beta 1$ stimulation (Fig. 1A). In addition, we assessed the effects of the exogenous TGF- $\beta 1$ treatment on the ability of HNSCC cells to induce wound closure to determine the biological relevance of the EMT-induced tumorigenicity in HNSCC cells. HNSCC cells migrated to an artificially produced wound in the culture dish to a markedly greater extent in the presence of TGF- $\beta 1$ in both HNSCC cell lines (Fig. 1B and C).

Induction of CD147 expression during TGF- $\beta 1$-induced EMT in HNSCC cells. In addition, we assessed the expression of EMT markers in two HNSCC cell lines cultured with TGF- $\beta 1$ to investigate whether these morphological changes and cell migration are the result of the EMT. We examined the expression of EMT markers, E-cadherin, and vimentin using western blot analysis. Although E-cadherin was downregulated, vimentin was upregulated with the TGF- $\beta 1$ treatment in both cell lines. These findings indicated that the EMT was induced in HNSCC cells by the TGF- $\beta 1$ treatment resulted in these morphological changes and cell migration. Interestingly, the CD147 expression was also upregulated by the TGF- $\beta 1$ stimulation (Fig. 2), suggesting that CD147 is correlated with the TGF- $\beta 1$-induced EMT in HNSCC cells. 
A

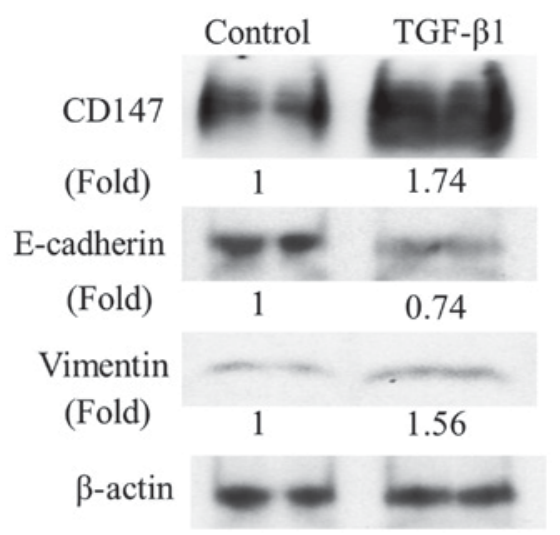

B

$\mathrm{FaDu}$

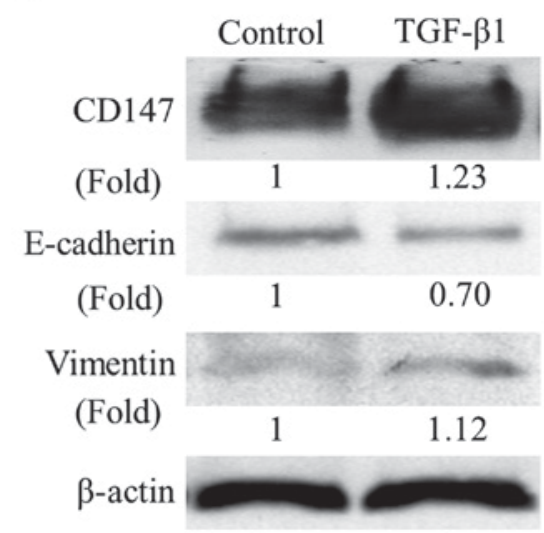

Figure 2. TGF- $\beta 1$ induces EMT and expression of CD147 in HNSCC cells. The expression of EMT markers, E-cadherin, and vimentin was examined by western blot analysis. $\beta$-actin was used as a loading control. E-cadherin was downregulated, and vimentin was upregulated in the TGF- $\beta 1$ treatment of (A) SAS and (B) FaDu cells. Meanwhile, the CD147 expression was also upregulated by the TGF- $\beta 1$ stimulation. SAS and FaDu cells were treated with $10 \mathrm{ng} / \mathrm{ml}$ of TGF- $\beta$ for $48 \mathrm{~h}$. The experiment was repeated thrice with similar results. EMT, epithelial-mesenchymal transition; TGF- $\beta 1$, transforming growth factor- $\beta 1$; HNSCC, head and neck squamous cell carcinoma.

A

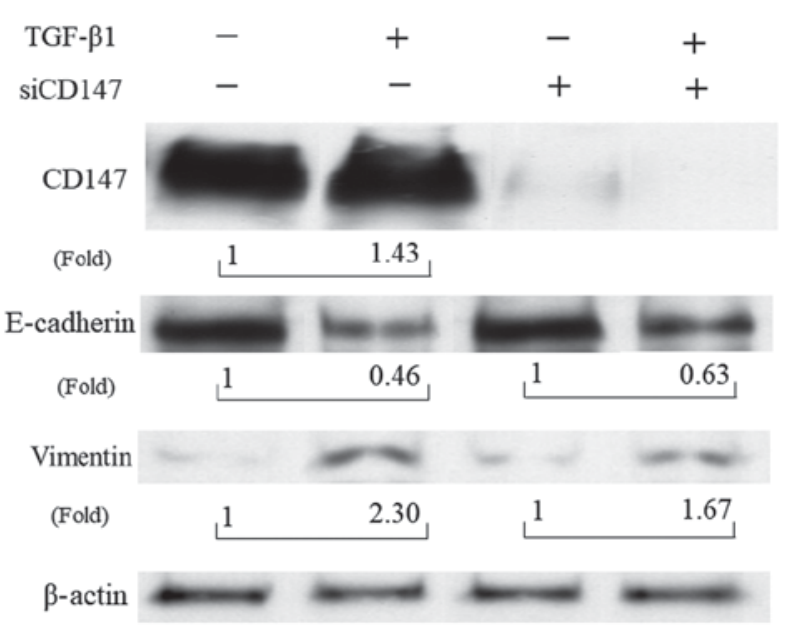

B

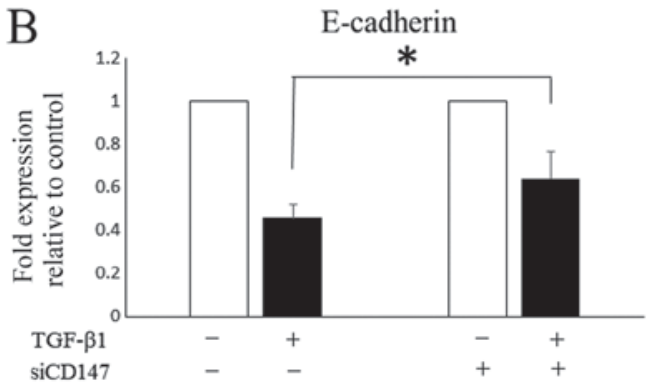

Vimentin

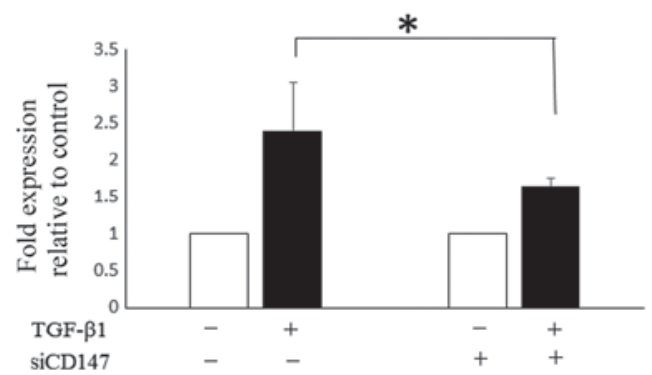

Figure 3. CD147 mediates the transforming growth factor- $\beta$ (TGF- $\beta$ )-induced EMT. SAS cells were transfected with nontargeting siRNA or CD147 siRNA and cultured with or without $10 \mathrm{ng} / \mathrm{ml}$ of TGF- $\beta 1$. (A) The expression of the epithelial protein E-cadherin and the mesenchymal protein vimentin was examined by western blot analysis. The experiment was repeated thrice with similar results. (B) The results are expressed as fold change relative to SAS cells without the TGF- $\beta 1$ stimulation. A fold decrease of E-cadherin and a fold increase of vimentin induced by the TGF- $\beta 1$ treatment were observed with both conditions of the CD147 knockdown or not; however, these changes were attenuated by the CD147 knockdown. The experiment was repeated thrice and the data shown are the means of three measurements, and the bars represent the $\mathrm{SD}$ of the mean. ${ }^{*} \mathrm{P}<0.05$. EMT, epithelial-mesenchymal transition; siRNA, small interfering RNA; TGF- $\beta 1$, transforming growth factor- $\beta 1$.

CD147 mediates the TGF- $\beta 1$-induced EMT and the following cell invasion of SCC cells of the tongue. The results suggest that TGF- $\beta 1$ induced the EMT in HNSCC cells (Fig. 1). In addition, the CD147 expression was upregulated during this TGF- $\beta 1$ induced EMT, suggesting that CD147 plays an important role in the TGF- $\beta 1$-induced EMT in HNSCC. Although CD147 is known to mediate the EMT in some solid tumors, it remains unclear whether CD147 mediates the EMT in HNSCC cells. Thus, we used siRNA on CD147 before culturing cells in the presence or absence of TGF- $\beta 1$ with SAS to validate whether CD147 mediates the EMT in HNSCC cells. The downregulation of E-cadherin and upregulation of vimentin by TGF- $\beta 1$ were attenuated when CD147 was silenced by siRNA (Fig. 3), indicating that CD147 partly contributed to the TGF- $\beta 1$-induced EMT in HNSCC cells.

Furthermore, on the basis of these findings, we hypothesized that CD147 mediates EMT-related malignant phenotypes in HNSCC. Thus, we analyzed the cell invasiveness of SAS in the presence or absence of siRNA-targeting CD147, with or without TGF- $\beta 1$, in the cell culture media to investigate the significance of CD147 in the tumorigenic process which induced by EMT. The findings revealed a trend for SAS to 
A

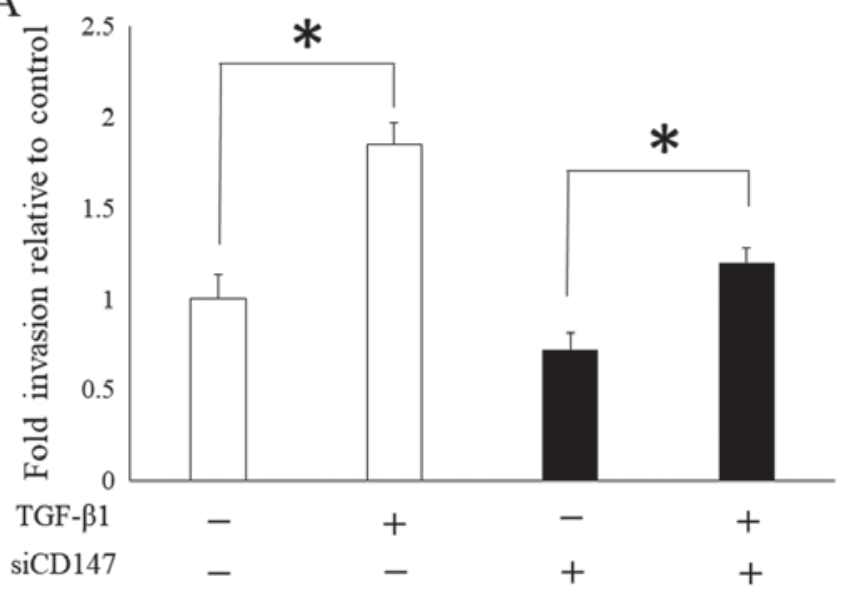

$\mathrm{B}$

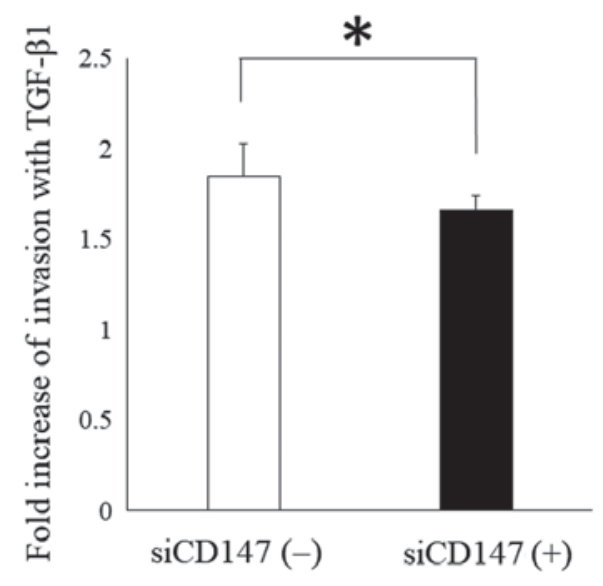

Figure 4. CD147 serves a vital role in TGF-)-induced cell invasion. SAS cells were transfected with nontargeting siRNA or CD147 siRNA, and cell invasion assay was performed using $10 \mathrm{ng} / \mathrm{ml}$ of TGF- $\beta 1$. (A) The results are expressed as fold change relative to SAS cells without TGF- $\beta 1$ and CD147 knockdown. Cell invasion increases with TGF- $\beta 1$ in either condition of the CD147 knockdown or not. (B) A fold increase of TGF- $\beta 1$-induced invasion was attenuated by the CD147 knockdown. The experiment was repeated thrice and the data shown are the means of three measurements, and the bars represent the SD of the mean. ${ }^{*} \mathrm{P}<0.05$. siRNA, small interfering RNA TGF- $\beta 1$, transforming growth factor- $\beta 1$; siRNA, small interfering RNA.

increase invasion with TGF- $\beta 1$ in either the presence or absence of CD147 knockdown (Fig. 4A). However, the fold increase in invasion was markedly lower in the presence of CD147 knockdown (Fig. 4B), suggesting that CD147 plays a vital role in cell invasion induced by the EMT in SAS.

\section{Discussion}

The present study confirmed that TGF- $\beta 1$ induces EMT following migration and CD147 is upregulated during TGF- $\beta 1$-induced EMT in HNSCC cell lines. In addition, CD147 knockdown attenuated this TGF- $\beta 1$-induced EMT and invasion in SCC of the tongue. These findings suggest that CD147 partly promotes EMT and cell invasion induced by the TGF- $\beta 1$ stimulation in individuals with HNSCC and indicated the need for further assessment of the mechanism underlying CD147 targeting HNSCC cells that express increased levels of such proteins. EMT is a crucial developmental process that often initiates during cancer invasion and metastasis (2). The molecular characteristics of EMT include the downregulation of epithelial cell markers and the upregulation of mesenchymal markers (14). Although various growth factors and cytokines can induce EMT, TGF- $\beta$ has a vital role in EMT (15); therefore, the mechanisms underlying the TGF- $\beta$-induced EMT must be further investigated to help identify novel, effective therapeutic strategies for cancer, including HNSCC.

The significance of CD147 in tumor progression and its correlation with poor prognosis have extensively been observed in individuals with solid tumors, which include those with HNSCC. Moreover, several studies have suggested that CD147 is a negative prognostic factor of malignant tumors (16). Previously, we have reported that the CD147-CD147 hemophilic interaction or CD147-cyclophilin A interaction has a vital role in matrix metalloproteinase expression and activation as well as invasion and migration $(8,11)$. In addition, other studies have shown a correlation between CD147 and EMT in several types of cancer $(9,13)$. However, data on the role of CD147 in the EMT in HNSCC are limited. The present study revealed that CD147 mediates the TGF- $\beta 1$-induced EMT in SCC of the tongue, which indicates that CD147 is an essential therapeutic target in HNSCC. Furthermore, studies have reported that $\mathrm{CD} 147$ has an antitumor effect against HNSCC (17). Additionally, we have previously reported that the inhibition of CD147 combined with the epidermal growth factor receptor increases the effect of growth prevention and migration of HNSCC cells (18). Therefore, more detailed knowledge regarding the role of CD147 in HNSCC progression might elucidate the prognosis of patients.

Recently, oncology and immunotherapy have been a topic of interest. Moreover, anti-PD-L1 therapies for recurrent or metastatic HNSCC have shown promising results, and cumulative studies regarding immunotherapy in patients with HNSCC have been conducted (19). Conversely, immunotherapy resistance is frequently observed in several patients with malignant tumors, including HNSCC (20). A recent study has reported that patients with $\mathrm{PD}$-1-therapy-resistant melanoma presented with distinct signatures of upregulated genes involved in immunosuppression and EMT (21). Moreover, EMT contributes to immunoescape and immunosuppression in individuals with solid tumors (22). Therefore, targeting these pathways, including EMT, via immunotherapy might help prevent resistance. In the present study, CD147 was found to mediate EMT in individuals with SCC of the tongue. CD147, which regulates EMT, might have an important role in improving the therapeutic effect of immunotherapy in individuals with HNSCC. However, our result cannot be generalized, and further studies must be conducted to validate the mechanisms underlying immunotherapy resistance in individuals with HNSCC.

In conclusion, CD147 plays a role in EMT and related tumorigenicity in SCC of the tongue. Furthermore, targeting CD147 may improve the prognoses of individuals with HNSCC.

\section{Acknowledgements}

Not applicable. 


\section{Funding}

The present study was supported by Grant-in-Aid for Scientific Research (C) (grant no. 15K10796) from the Ministry of Education, Culture, Sports, Science and Technology, Japan.

\section{Availability of data and materials}

The datasets used and analyzed during the current study are available from the corresponding author on reasonable request.

\section{Authors' contributions}

SS conceived and designed the experiments. SS, ST, TT and YK performed the experiments. SS analyzed data and contributed to writing of the manuscript. TY performed experiments, and data analysis and interpretation. All authors reviewed the final manuscript.

\section{Ethics approval and consent to publication}

Not applicable.

\section{Patient consent for publication}

Not applicable.

\section{Competing interests}

The authors declare that they have no competing interests.

\section{References}

1. Cho J, Johnson DE and Grandis JR: Therapeutic implications of the genetic landscape of head and neck cancer. Semin Radiat Oncol 28: 2-11, 2018.

2. KalluriR and Weinberg RA: The basics of epithelial-mesenchymal transition. J Clin Invest 119: 1420-1428, 2009.

3. Wendt MK, Allington TM and Schiemann WP: Mechanisms of the epithelial-mesenchymal transition by TGF-beta. Futur Oncol 5: 1145-1168, 2009.

4. Peinado H, Olmeda D and Cano A: Snail, Zeb and bHLH factors in tumour progression: An alliance against the epithelial phenotype? Nat Rev Cancer 7: 415-428, 2007.

5. Miettinen PJ, Ebner R, Lopez AR and Derynck R: TGF-beta induced transdifferentiation of mammary epithelial cells to mesenchymal cells: Involvement of type I receptors. J Cell Biol 127: 2021-2036, 1994.

6. Smith A, Teknos TN and Pan Q: Epithelial to mesenchymal transition in head and neck squamous cell carcinoma. Oral Oncol 49: 287-292, 2013.

7. Caudroy S, Polette M, Tournier JM, Burlet H, Toole B, Zucker S and Birembaut P: Expression of the extracellular matrix metalloproteinase inducer (EMMPRIN) and the matrix metalloproteinase-2 in bronchopulmonary and breast lesions. J Histochem Cytochem 47: 1575-1580, 1999.
8. Suzuki S, Sato M, Senoo H and Ishikawa K: Direct cell-cell interaction enhances pro-MMP-2 production and activation in co-culture of laryngeal cancer cells and fibroblast: Involvement of EMMPRIN and MT1-MMP. Exp Cell Res 293: 259-266, 2004.

9. Xu T, Zhou M, Peng L, Kong S, Miao R, Shi Y, Sheng H and Li L: Upregulation of CD147 promotes cell invasion, epithelial-to-mesenchymal transition and activates MAPK/ERK signaling pathway in c. Int J Clin Exp Pathol 7: 7432-7441, 2014.

10. Hibino T, Sakaguchi M, Miyamoto S, Yamamoto M, Motoyama A, Hosoi J, Shimokata T, Ito T, Tsuboi R and Huh NH: S100A9 is a novel ligand of EMMPRIN that promotes melanoma metastasis. Cancer Res 73: 172-183, 2013.

11. Takahashi M, Suzuki S and Ishikawa K: Cyclophilin A-EMMPRIN interaction induces invasion of head and neck squamous cell carcinoma. Oncol Rep 27: 198-203, 2012.

12. Suzuki S, Honda K, Nanjo H, Iikawa N, Tsuji T, Kawasaki Y, Yamazaki K, Sato T, Saito H, Shiina K and Ishikawa K: CD147 expression correlates with lymph node metastasis in T1-T2 squamous cell carcinoma of the tongue. Oncol Lett 14: 4670-4676, 2017.

13. Ru NY, Wu J, Chen ZN and Bian H: HAb18G/CD147 is involved in TGF- $\beta$-induced epithelial-mesenchymal transition and hepatocellular carcinoma invasion. Cell Biol Int 39: 44-51, 2015.

14. Liu F, Gu LN, Shan BE, Geng CZ and Sang MX: Biomarkers for EMT and MET in breast cancer: An update. Oncol Lett 12: 4869-4876, 2016.

15. Katsuno Y, Lamouille S and Derynck R: TGF- $\beta$ signaling and epithelial-mesenchymal transition in cancer progression. Curr Opin Oncol 25: 76-84, 2013.

16. Zhu C, Pan Y, He B, Wang B, Xu Y, Qu L, Bao Q, Tian F and Wang S: Inhibition of CD147 gene expression via RNA interference reduces tumor cell invasion, tumorigenicity and increases chemosensitivity to cisplatin in laryngeal carcinoma Hep2 cells. Oncol Rep 25: 425-432, 2011.

17. Ma C, Wang J, Fan L and Guo Y: Inhibition of CD147 expression promotes chemosensitivity in HNSCC cells by deactivating MAPK/ERK signaling pathway. Exp Mol Pathol 102: 59-64, 2017.

18. Suzuki S and Ishikawa K: Combined inhibition of EMMPRIN and epidermal growth factor receptor prevents the growth and migration of head and neck squamous cell carcinoma cells. Int J Oncol 44: 912-917, 2014.

19. Ferris RL, Blumenschein G Jr, Fayette J, Guigay J, Colevas AD, Licitra L, Harrington KJ, Kasper S, Vokes EE, Even C, et al: Nivolumab vs. investigator's choice in recurrent or metastatic squamous cell carcinoma of the head and neck: 2-year long-term survival update of CheckMate 141 with analyses by tumor PD-L1 expression. Oral Oncol 81: 45-51, 2018.

20. Kelderman S, Schumacher TN and Haanen JB: Acquired and intrinsic resistance in cancer immunotherapy. Mol Oncol 8: 1132-1139, 2014

21. Bu X, Mahoney KM and Freeman GJ: Learning from PD-1 resistance: New combination strategies. Trends Mol Med 22: 448-451, 2016.

22. Terry S, Savagner P, Ortiz-Cuaran S, Mahjoubi L, Saintigny P, Thiery JP and Chouaib S: New insights into the role of EMT in tumor immune escape. Mol Oncol 11: 824-846, 2017.

(i) $(-)$ This work is licensed under a Creative Commons Attribution-NonCommercial-NoDerivatives 4.0 International (CC BY-NC-ND 4.0) License. 